\title{
Transcriptome-wide identification of mRNAs and lincRNAs associated with trastuzumab-resistance in HER2-positive breast cancer
}

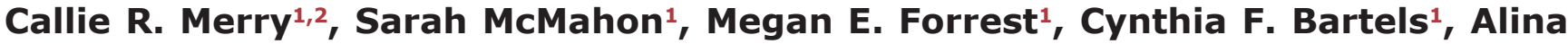 \\ Saiakhova $^{1}$, Courtney A. Bartel ${ }^{3}$, Peter C. Scacheri ${ }^{1,3}$, Cheryl L. Thompson ${ }^{3,5}$, Mark \\ W. Jackson3 ${ }^{3}$ Lyndsay N. Harris ${ }^{3,4}$, Ahmad M. Khalil1,2,3 \\ ${ }^{1}$ Department of Genetics and Genome Sciences, Case Western Reserve University, Cleveland, OH 44106, USA \\ ${ }^{2}$ Department of Biochemistry, Case Western Reserve University, Cleveland, OH 44106, USA \\ ${ }^{3}$ Case Comprehensive Cancer Center, Case Western Reserve University, Cleveland, OH 44106, USA \\ ${ }^{4}$ Department of Medicine and Case Western Reserve University, Cleveland, OH 44106, USA \\ ${ }^{5}$ Department of Nutrition, Case Western Reserve University, Cleveland, OH 44106, USA \\ Correspondence to: Ahmad M. Khalil, email: Dr.Ahmad.Khalil@gmail.com \\ Keywords: breast cancer, HER2, drug resistance, trastuzumab-resistance, cancer therapy \\ Received: March 11, 2016 \\ Accepted: July 09, 2016 \\ Published: July 16, 2016
}

\section{ABSTRACT}

Approximately, 25-30\% of early-stage breast tumors are classified at the molecular level as HER2-positive, which is an aggressive subtype of breast cancer. Amplification of the HER2 gene in these tumors results in a substantial increase in HER2 MRNA levels, and consequently, HER2 protein levels. HER2, a transmembrane receptor tyrosine kinase (RTK), is targeted therapeutically by a monoclonal antibody, trastuzumab ( $\mathrm{Tz}$ ), which has dramatically improved the prognosis of HER2-driven breast cancers. However, $\sim 30 \%$ of patients develop resistance to trastuzumab and recur; and nearly all patients with advanced disease develop resistance over time and succumb to the disease. Mechanisms of trastuzumab resistance (TzR) are not well understood, although some studies suggest that growth factor signaling through other receptors may be responsible. However, these studies were based on cell culture models of the disease, and thus, it is not known which pathways are driving the resistance in vivo. Using an integrative transcriptomic approach of RNA isolated from trastuzumab-sensitive and trastuzumab-resistant HER2+ tumors, and isogenic cell culture models, we identified a small set of mRNAs and lincRNAs that are associated with trastuzumab-resistance (TzR). Functional analysis of a top candidate gene, S100P, demonstrated that inhibition of S100P results in reversing TzR. Mechanistically, S100P activates the RAS/MEK/MAPK pathway to compensate for HER2 inhibition by trastuzumab. Finally, we demonstrated that the upregulation of S100P appears to be driven by epigenomic changes at the enhancer level. Our current findings should pave the path toward new therapies for breast cancer patients.

\section{INTRODUCTION}

Breast cancer is a major health problem affecting millions of patients worldwide, and results in over 500,000 deaths annually. Previous studies have led to the classification of breast tumors into several molecular subtypes, with HER2-positive (HER2+) tumors representing $\sim 25-30 \%$ of early-stage breast cancer patients' diagnoses [1-3]. HER2+ tumors are characterized at the molecular level by an amplification of a genomic region encompassing the HER2 gene (also known as HER2/neu and ERBB2), which is a member of the ERBB family of transmembrane receptor tyrosine kinases (RTKs) [1]. Although no known ligands bind to the HER2 receptor itself, a number of ligands bind to other ERBB family members (e.g., HER3), and lead to the heterodimerization of these members with HER2 [4]. Heterodimerization results in autophosphorylation by the intracellular tyrosine kinase domain of HER2, and the initiation of a signaling cascade that results in the activation and repression of 
specific mRNAs and long intergenic non-coding RNAs (lincRNAs) [5].

Trastuzumab $\left(\right.$ Herceptin $\left.^{\circledR}\right)$ is a monoclonal antibody that binds to the extracellular domain of the HER2 receptor, and is thought to inhibit its heterodimerization, and consequently, its signaling cascade [6]. Currently, trastuzumab is the standard of care for HER $2+$ breast cancer patients as $70 \%$ of early-stage patients appear to be cured by trastuzumab and chemotherapy. However, a significant percentage of early-stage patients $(\sim 30 \%)$ relapse after this combination by unclear mechanisms [7]. Furthermore, most patients who relapse will acquire resistance to trastuzumab during therapy and succumb to the disease $[6,8,9]$. Although several mechanisms of TzR have been proposed, it has been difficult to show convincingly that these occur in the clinical setting $[6,9]$. For example, it was hypothesized that resistance occurs due to shedding of the extracellular domain of HER2, and thus, trastuzumab is no longer able to bind to HER2 [10], however, no experimental evidence has been presented to support this. Other models proposed that HER3 signaling could compensate for HER2 inhibition by trastuzumab. However, patients who received a combination of trastuzumab and pertuzumab, which blocks HER2-HER3 interaction, have a median progression-free survival of 5.5 months [11]. Thus, it is clear that other mechanisms of resistance are at play in tumors in vivo.

Given the immense potential clinical benefits in identifying mechanisms of TzR, we decided to take a comprehensive and unbiased approach to identify the key cellular factors that drive TzR in humans in vivo. By applying next generation RNA-sequencing to RNA isolated from human HER2+ tumors and cell culture models of the disease that are either trastuzumab-sensitive (TzS) or trastuzumab-resistant (TzR), we have identified a small set of mRNAs and lincRNAs that are strongly associated with TzR (Figure 1A). Functional studies of a top candidate gene, S100P, demonstrated that inhibition of $\mathrm{S} 100 \mathrm{P}$ results in reversal of trastuzumab-resistance (TzR). Our study provides for the first time a clinically relevant mechanism of TzR, and opens the door for exploring novel therapeutic strategies.

\section{RESULTS}

\section{Identification of differentially expressed mRNAs and lincRNAs in trastuzumab-sensitive (TzS) vs trastuzumab-resistant (TzR) human tumors in vivo}

We hypothesized that TzR tumors have a distinct gene expression profile compared to TzS tumors, and a subset of these differentially expressed coding and noncoding genes mediate the observed resistance. To that end, we applied RNA-seq to RNA isolated from HER2+ tumor biopsies that were collected during a clinical trial (see methods). In this clinical trial, 50 patient tumors were biopsied at the beginning of the trial prior to receiving trastuzumab (pre-trastuzumab). After receiving a combination of chemotherapy and trastuzumab for 4 months, a subset of thirteen tumors, representing the extremes of response to treatment, were selected for gene expression analysis by RNA-seq. Eleven of those thirteen patients were identified as responders (i.e., trastuzumabsensitive or $\mathrm{TzS}$ ) and two patients were identified as non-responders (i.e., trastuzumab-resistant or TzR) as measured by pathological complete response (pCR) or clinical non-response (SD+PD). We compared mRNA gene expression between the responders (TzS) and nonresponders (TzR), and identified differentially expressed genes between the two groups prior to receiving treatment (pre-trastuzumab) (Figure 1A). Specifically, we identified $\sim 1,542$ mRNAs and 371 lincRNAs that are differentially expressed (Figure 1B, and Supplementary Files S1 and S2). We performed pathway analysis on differentially expressed mRNAs, and found several pathways to be affected including those related to mammary gland cell proliferation and development (Figure 1C). Also, RAGE receptor binding was statistically significant and emerged as a key player (see below). Currently, it is not possible to perform pathway analysis on lincRNAs as only a small percentage of all annotated lincRNAs $(\sim 10,000$ in the human genome) have been functionally characterized ( 200 lincRNAs) [12].

\section{Characterization of TzS and TzR HER2+ cell culture model}

To further pinpoint key mRNAs and lincRNAs that contribute to TzR, we generated TzR HER2+ BT474 cells by chronically exposing the cell line to trastuzumab over six weeks. To confirm that we have generated BT474 cells that are resistant to trastuzumab (TzR), we measured cell proliferation of the parental TzS and isogenic TzR cells under exposure to trastuzumab over a 96 hour time period. We found that the proliferation of parental TzS BT474 cells is significantly affected when exposed to trastuzumab within 48 hours. By contrast, the proliferation of TzR BT474 cells was not affected (Figure 2A-2B).

Next, we examined the effect of trastuzumab on HER2 and phospho-HER2 (p-HER2) protein levels in TzS and TzR cells. We found TzS cells to express comparable levels of HER2 protein to TzR cells (Figure 2C). However, trastuzumab strongly inhibits the phosphorylation of HER2 (p-HER2) in TzS cells within 24 hours of exposure (Figure 2D). Next, we examined the expression of HER2 and p-HER2 levels in both TzS and TzR cells over a 96-hour time course, and found: i. HER2 protein levels are not significantly affected in the parental TzS line by trastuzumab until the 96-hour time point (Figure 2E), ii. p-HER2 levels significantly decrease in the parental TzS line within 12 hours post-exposure to trastuzumab 
(Figure 2F), iii. Surprisingly, both HER2 and p-HER2 levels are significantly affected in the TzR BT474 cells at 48 hours post exposure to trastuzumab (Figure $2 \mathrm{G}-2 \mathrm{H}$ ). The decrease in HER2 and p-HER2 levels in the TzR line demonstrates that trastuzumab can still bind to and inhibit HER2 phosphorylation in TzR cells. These observations demonstrate that the mechanism of TzR, at least in a subset of tumors, is not due to truncation or mutations of HER2 that precludes trastuzumab binding.
Transcriptome-wide changes in gene expression in a cell culture model of $\mathrm{TzR}$

To identify genes associated with TzR in our cell culture model described above, we quantified changes in gene expression between parental TzS BT474 and TzR BT474 cells by RNA-seq. We identified 233 mRNAs and 34 lincRNAs as differentially expressed (fold change $\geq 2$, $p \leq 0.05)$ (Figure 3A-3B, Supplementary Files S3 and S4).
A

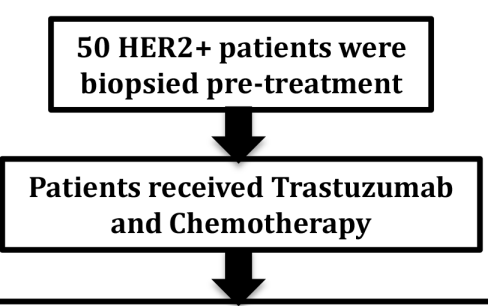

Responders and non-responders are identified at end of trial, pCR $(p<0.05)$

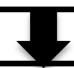

Biopsies (pre-treatment) from 11 responders and 2 non-responders were utilized for RNA-seq

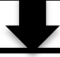

Differential gene expression analysis, and intersection with differentially expressed genes identified in cell culture models (i.e., TzS vs TzR isogenic cell lines)

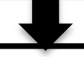

Functional studies of top candidates (i.e., reversing trastuzumab resistance)
B

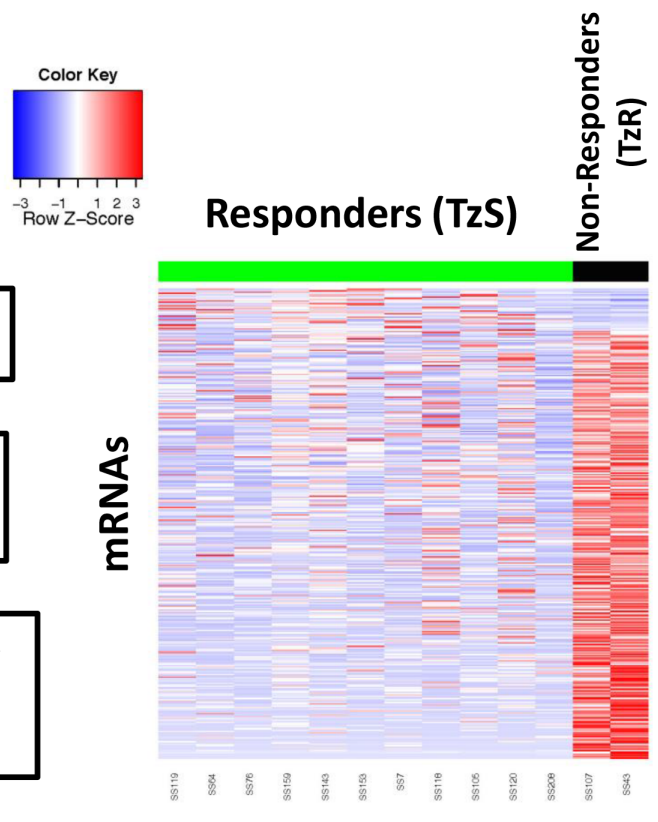

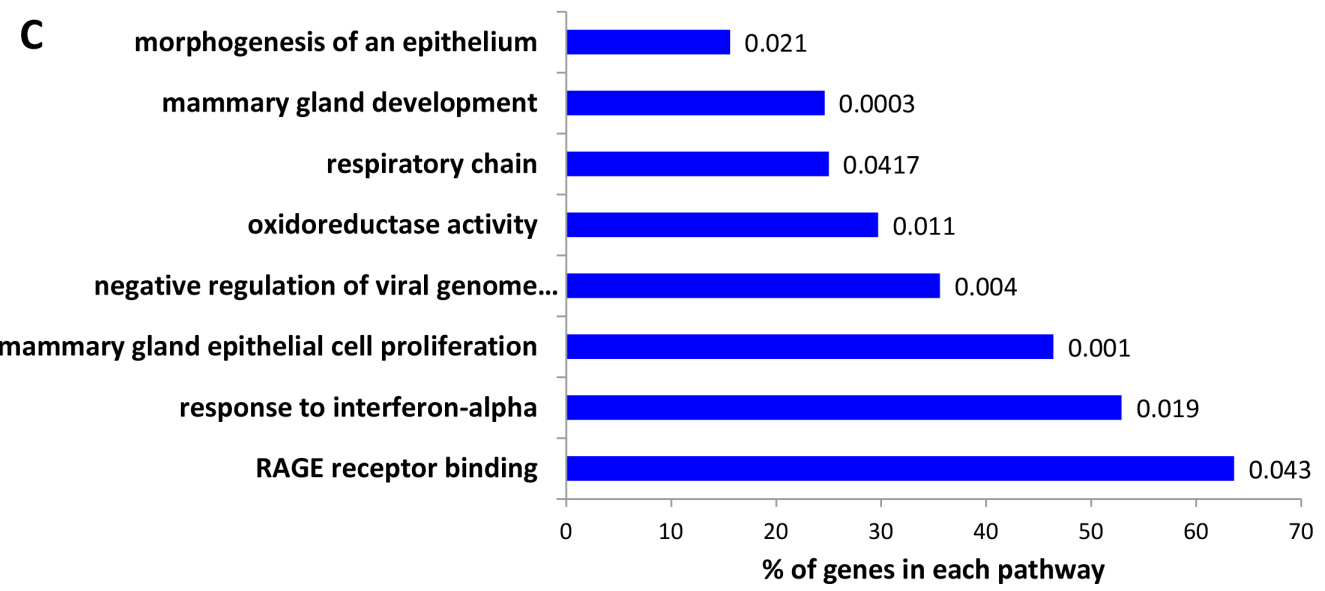

Figure 1: Identification of differentially expressed genes in trastuzumab-resistant (TzR) vs trastuzumab-sensitive (TzS) tumors by RNA-seq. (A) A schematic outlining overall study design. Differentially expressed genes identified in TzS vs TzR human HER2+ tumors in vivo were intersected with differentially expressed genes identified in TzS vs TzR BT474 cell lines, which led to a small list of mRNAs and lincRNAs that are associated with TzR. (B) Heatmap representation of the differentially expressed mRNAs in TzS patients (responders) vs TzR patients (non-responders). (C) Pathway analysis of differentially expressed mRNAs in tumors in vivo. Pathway names are listed on the Y-axis, the percentage of genes affected in each pathway is indicated on the $\mathrm{X}$-axis, and a $p$-value is given at the end of each bar. 
To further refine our list of genes that are specifically associated with TzR, and not due to short-term exposure to trastuzumab, we treated the parental TzS BT474 cells with trastuzumab, and collected RNA at 48 hours postexposure. We measured changes in gene expression by RNA-seq and identified 242 mRNAs and 27
lincRNAs that are differentially expressed as compared to mock treated cells $(\geq 2$ fold change and $p \leq 0.05$ ) (Supplementary Files S5 and S6). Subsequently, mRNAs and lincRNAs that are affected by short-term exposure to trastuzumab were subtracted from mRNAs and lincRNAs that are differentially expressed in TzR vs TzS cells.
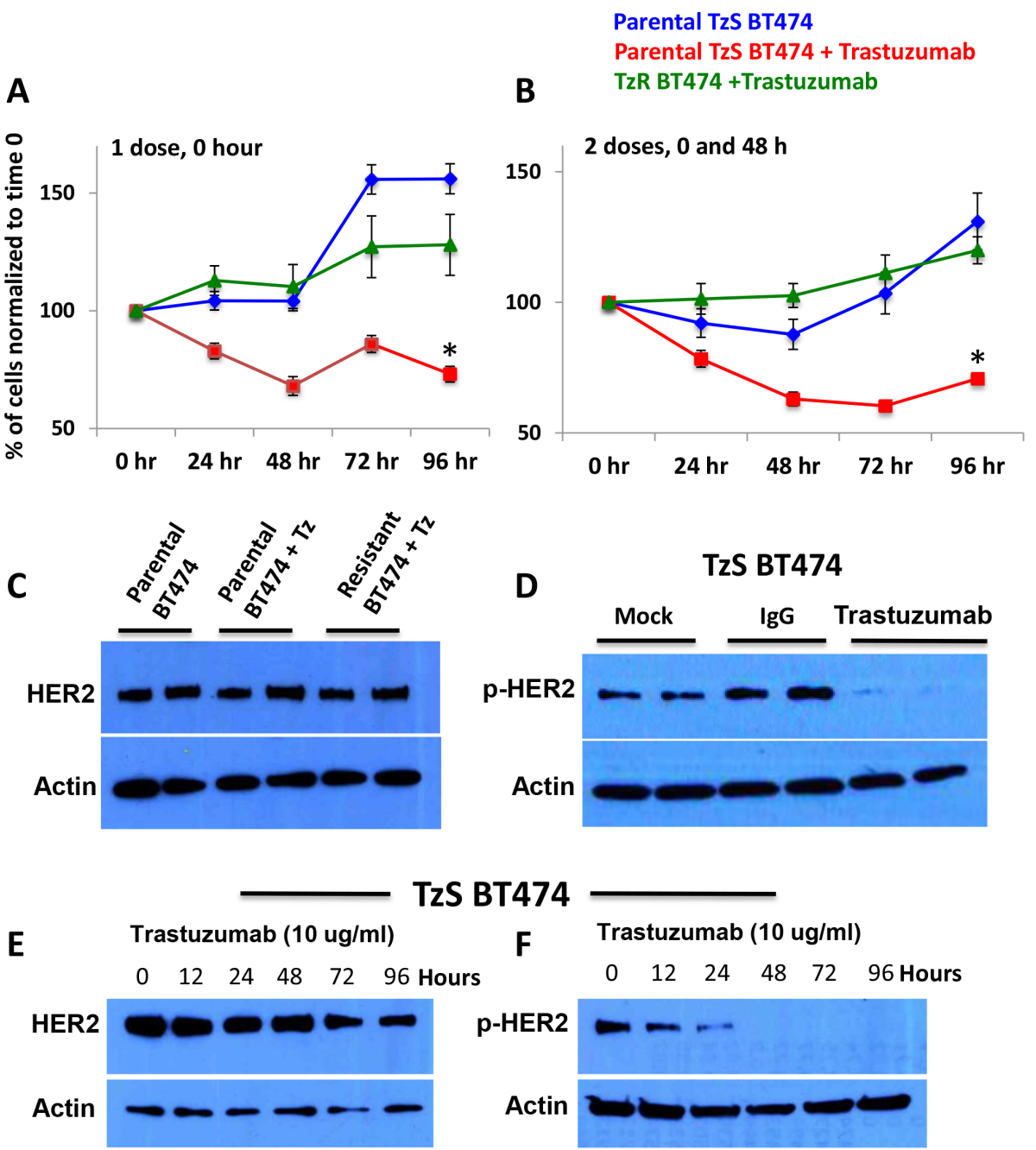

\section{TzS BT474}

F Trastuzumab $(10 \mathrm{ug} / \mathrm{ml})$
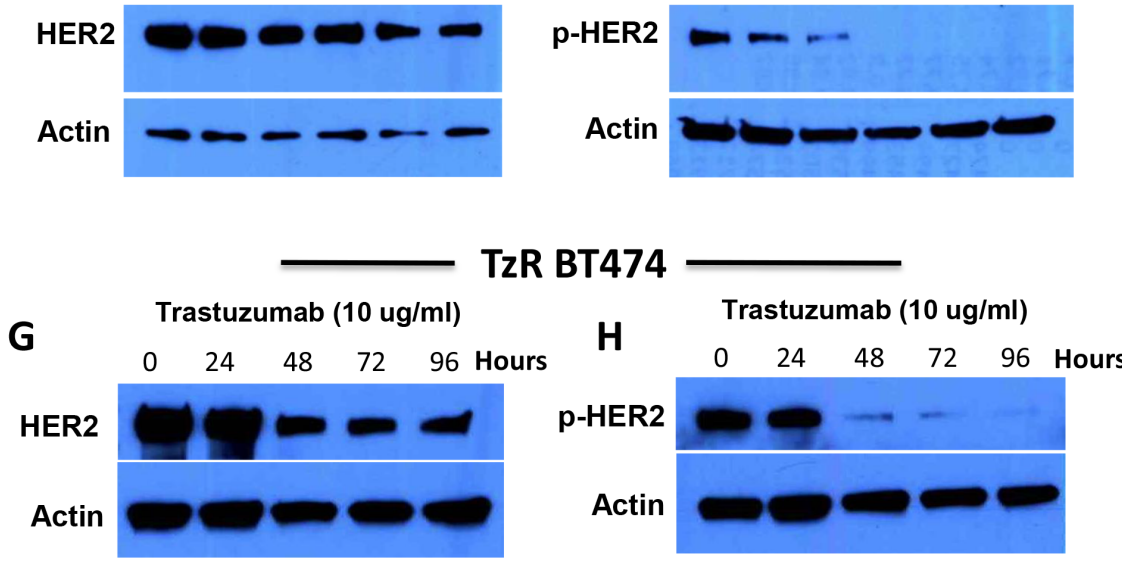

Figure 2: Characterization of HER2-positive trastuzumab-sensitive (TzS) and trastuzumab-resistant (TzR) BT474 breast cancer cell lines. (A-B) Cell proliferation analysis of TzS and TzR BT474 cell lines with either (A) one dose of $10 \mu \mathrm{g} / \mathrm{ml}$ trastuzumab at $0 \mathrm{hr}$ or (B) $10 \mu \mathrm{g} / \mathrm{ml}$ trastuzumab at 0 and 48 hours demonstrated that while TzS BT474 cells is significantly decreased by trastuzumab, TzR cells proliferation is not affected. (C) Western blot analysis demonstrates that both TzS and TzR BT474 cells have comparable levels of HER2 protein. (D) Trastuzumab $(10 \mu \mathrm{g} / \mathrm{ml})$ treatment significantly affects p-HER2 levels in TzS BT474 cells within 24 hours post treatment. (E) HER2 protein levels stay relatively constant in TzS BT474 cells with trastuzumab treatment over 96 hours. (F) p-HER2 levels decrease significantly at 24 hours post trastuzumab treatment, and remain undetectable at a $96 \mathrm{~h}$ timepoint. (G-H) Decreased levels of HER2 and p-HER2 levels are observed in TzR cells at $48 \mathrm{~h}$ post trastuzumab treatment. 

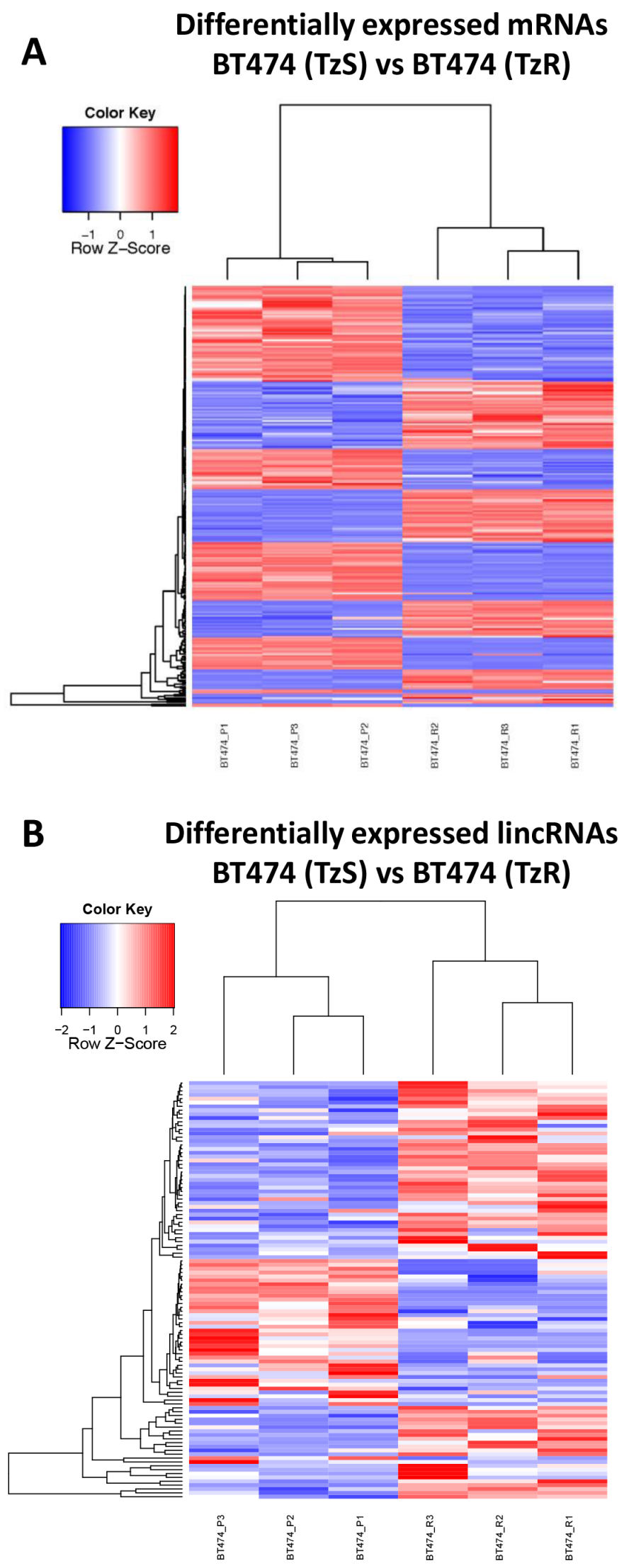

Figure 3: Numerous mRNAs and lincRNAs are differentially expressed in TzS vs TzR BT474 cells. Heatmap representation of differentially expressed (A) mRNAs and (B) lincRNAs in TzS BT474 vs TzR BT474 isogenic cell lines. 
This enabled us to further pinpoint mRNAs and lincRNAs that are specifically associated with long-term resistance to trastuzumab in culture. This analysis narrowed down the gene list to $201 \mathrm{mRNAs}$ and 25 lincRNAs.

We intersected mRNAs and lincRNAs identified in our cell culture model with differentially expressed mRNAs and lincRNAs identified in our in vivo data set (clinical trial). This analysis led to the identification of 18 mRNAs and 7 lincRNAs that are associated with TzR in vivo and in cell culture, with concordant expression patterns ( $p<0.0001$, two-tailed Fischer's exact test) (Supplementary File S7). Expression of top mRNA genes identified in our analysis in TzS vs TzR tumors in vivo is shown using cluster graphs, HER2 expression is also shown to demonstrate that HER2 levels do not change dramatically between $\mathrm{TzS}$ and TzR tumors (Figure 4). Careful examination of these genes, their known roles in breast cancer, and our bioinformatics analysis of key pathways associated with TzR in vivo (see Figure 1C), suggested a potential key role of
S100P. S100P is part of the S100 protein family, has documented roles in tumorigenesis [13], and is known to bind to the receptor RAGE (see Figure 1C), and activates similar signaling pathways to those activated by HER2 signaling. Furthermore, analysis of S100P in Oncomine demonstrated that $\mathrm{S} 100 \mathrm{P}$ is strongly associated with breast cancer ( $p=1.8 \mathrm{E}-94$, average fold change $=10.9$ ) (Supplementary Figure S1). Lastly, examination of RNA-seq of The Cancer Genome Atlas (TCGA) also demonstrated that S100P is highly upregulated in HER2+ breast cancer (Figure 5A).

To further confirm the upregulation of S100P in TzR cells, we examined S100P mRNA and protein levels in the TzS vs TzR BT474 cell line utilized for our RNAseq studies, and two independently generated $\mathrm{TzS}$ vs TzR BT474 and SKBR3 cell lines (three isogenic TzS vs TzR cell lines total). We found S100P to be upregulated at both the mRNA (Figure 5B) and protein levels (Figure 5C), with potentially increased stability of the S100P mRNA in SKBR3 cells. Importantly, we found S100P to be

\section{TzS Tumors TzR Tumors}

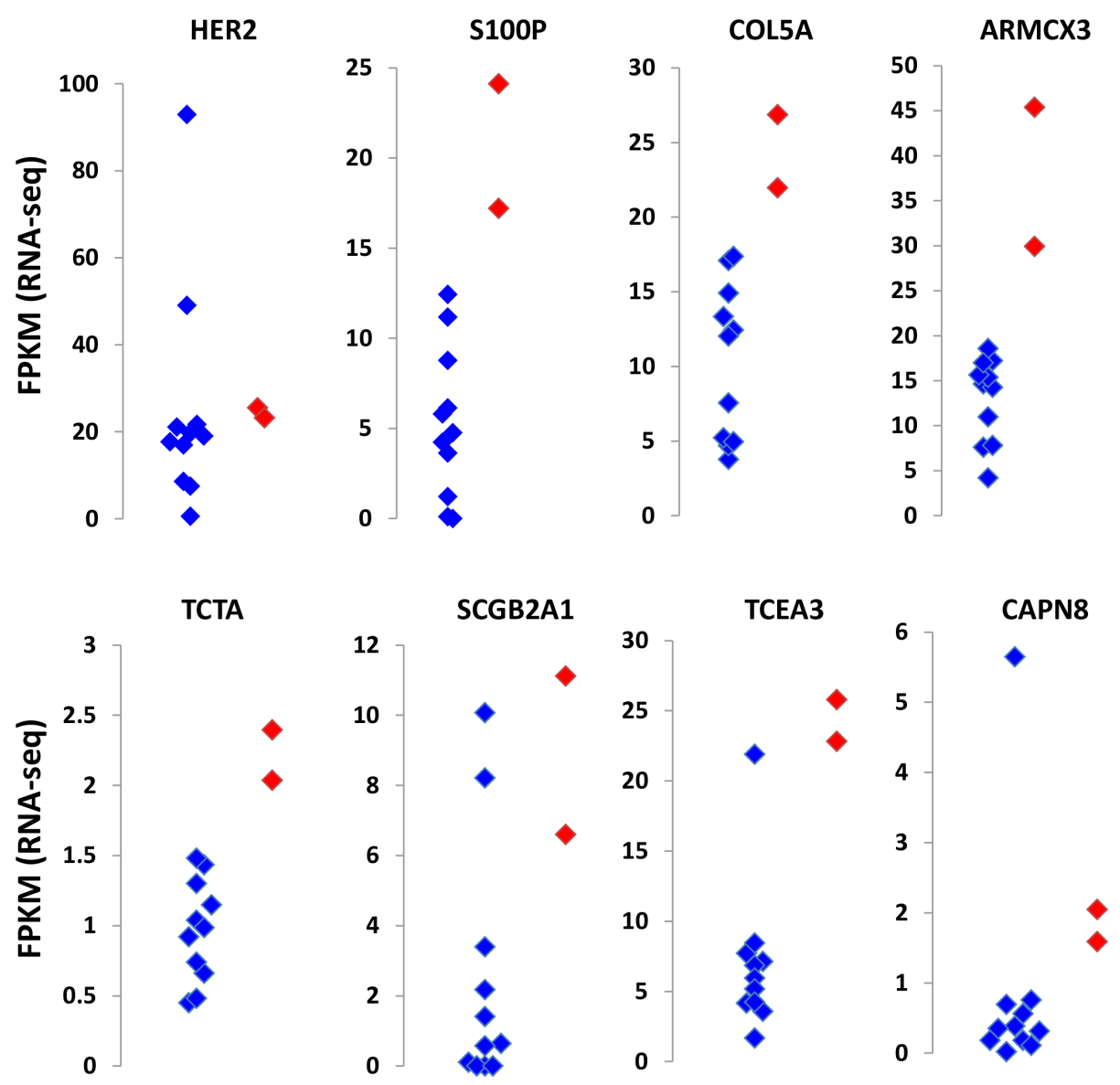

Figure 4: Top candidate mRNAs associated with trastuzumab-resistant (TzR). The expression of each mRNA in each tumor sample (blue dots: TzS, red dots: TzR) is shown as FPKM value based on RNA-seq analysis. HER2 mRNA levels are shown as a control and do not significantly change between TzS vs TzR tumors. By contrast, mRNAs identified in our analysis show striking upregulation in TzR tumors (red) vs the majority of TzS tumors (blue). 
upregulated in a cohort of breast cancer cell lines as compared to normal human mammary epithelial cells (Supplementary Figure S2), demonstrating that S100P is upregulated from normal tissues to breast tumors, and further upregulated or stabilized in TzR tumors.

\section{Inhibition of S100P reverses $\mathrm{TzR}$ in a cell culture model}

To test the potential role of $\mathrm{S} 100 \mathrm{P}$ in TzR, we designed and tested five distinct shRNAs against S100P, and identified two shRNAs that are effective at knocking down S100P (Supplementary Figure S3A). To determine if the depletion of S100P would re-sensitize TzR cells to trastuzumab, we tested the proliferation of TzR BT474 cells that were infected with either shGFP or shS100P in mock vs trastuzumab treatments. BT474 cells infected with shGFP showed no response to trastuzumab (Figure 6A), by contrast, the proliferation of TzR BT474 cells infected with shS100P was significantly impacted when exposed to trastuzumab (Figure 6B-6C). We also examined the rate of apoptosis of TzR cells in response to shRNA-mediated depletion of S100P, and found a significant increase in apoptosis in TzR cells treated with shS100P vs shGFP (Supplementary Figure S4). Lastly, we performed soft agar colony formation assays under trastuzumab treatment, and found that the knock down of S100P results in a significant decrease in number of colonies, as compared to shGFP (Figure 7A). Collectively, these data demonstrate that depletion of S100P partially resensitizes TzR cells to trastuzumab.
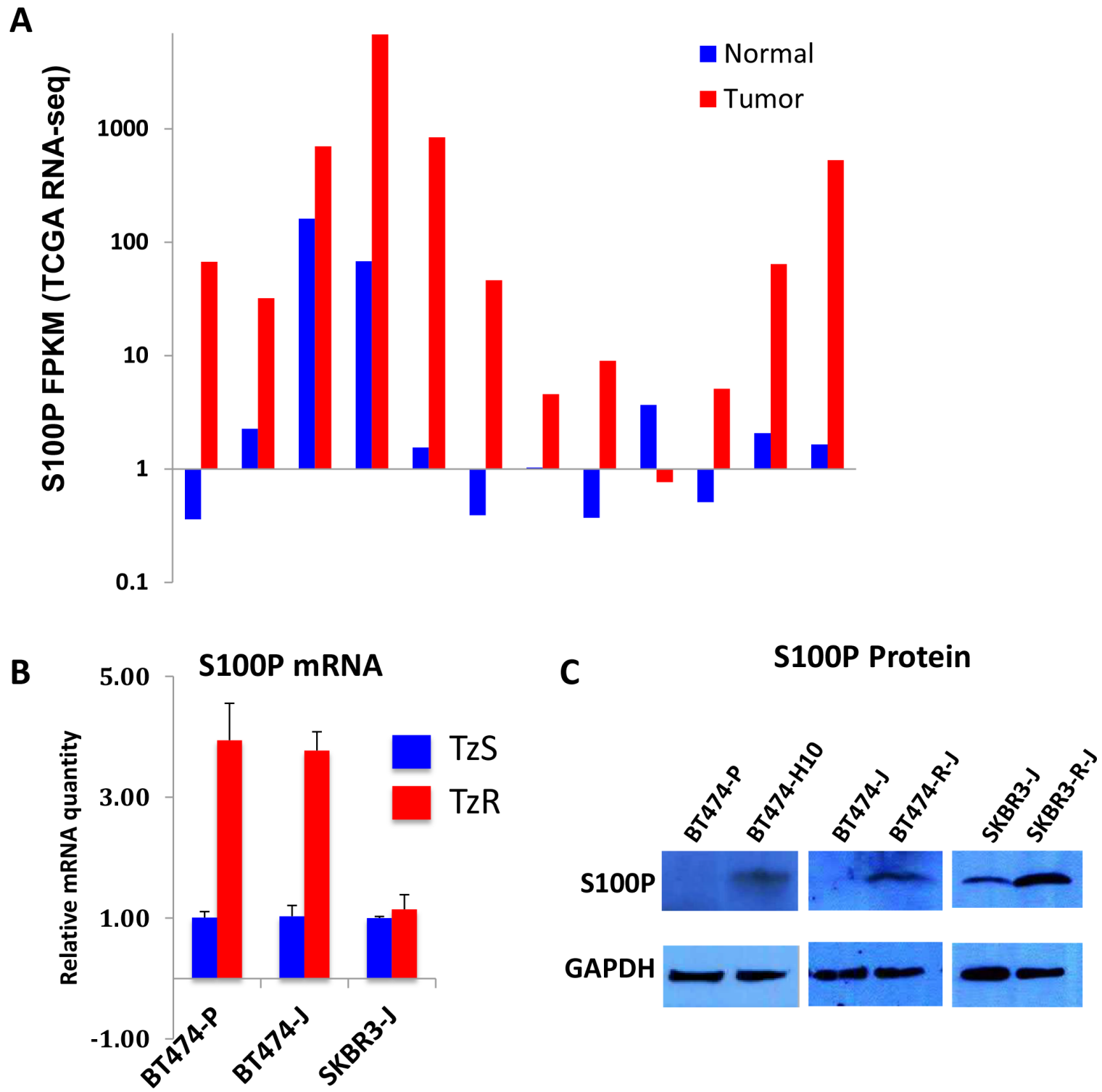

Figure 5: S100P is upregulated in trastuzumab-resistant cells. (A) S100P is highly upregualted in HER2+ tumors as compared to matched normal breast tissues (TCGA RNA-seq data). (B) S100P mRNA is elevated in two independently generated TzR vs TzS BT474 cells. (C) S100P protein is elevated in TzR vs TzS BT474 and SKBR3 cells as measured by western blot analysis. 


\section{S100P activates Ras/MEK/MAPK pathway}

S100P is known to bind to the receptor RAGE, leading to activation of the Ras/MEK/MAPK and other signaling pathways [14]. The Ras/MEK/MAPK is a key pathway that is known to be activated by HER2 signaling [1]. To test the hypothesis that S100P leads to activation of Ras/MEK/MAPK, and thus compensates for HER2 inhibition by trastuzumab, we performed the following experiments. First, we examined the effect of recombinant S100P on p-MAPK in TzS cells as follows: BT474 cells (TzS) were treated with trastuzumab alone or with trastuzumab and recombinant $\mathrm{S} 100 \mathrm{P}$ protein, and the levels of pMAPK, MAPK and histone H3 (control) were measured by WB. We found that trastuzumab treatment results in reducing p-MAPK levels, but the addition of $\mathrm{S} 100 \mathrm{P}$ recombinant protein is sufficient to restore p-MAPK protein levels (Figure 7B). Next, we examined the effect of knocking down S100P on p-MAPK in TzR cells. We found that TzR cells harboring shGFP had minor reduction of p-MAPK when treated with trastuzumab, however, TzR cells harboring shS100P showed significant reduction of p-MAPK when treated with trastuzumab (Figure 7C). These findings demonstrate that the upregulation of S100P

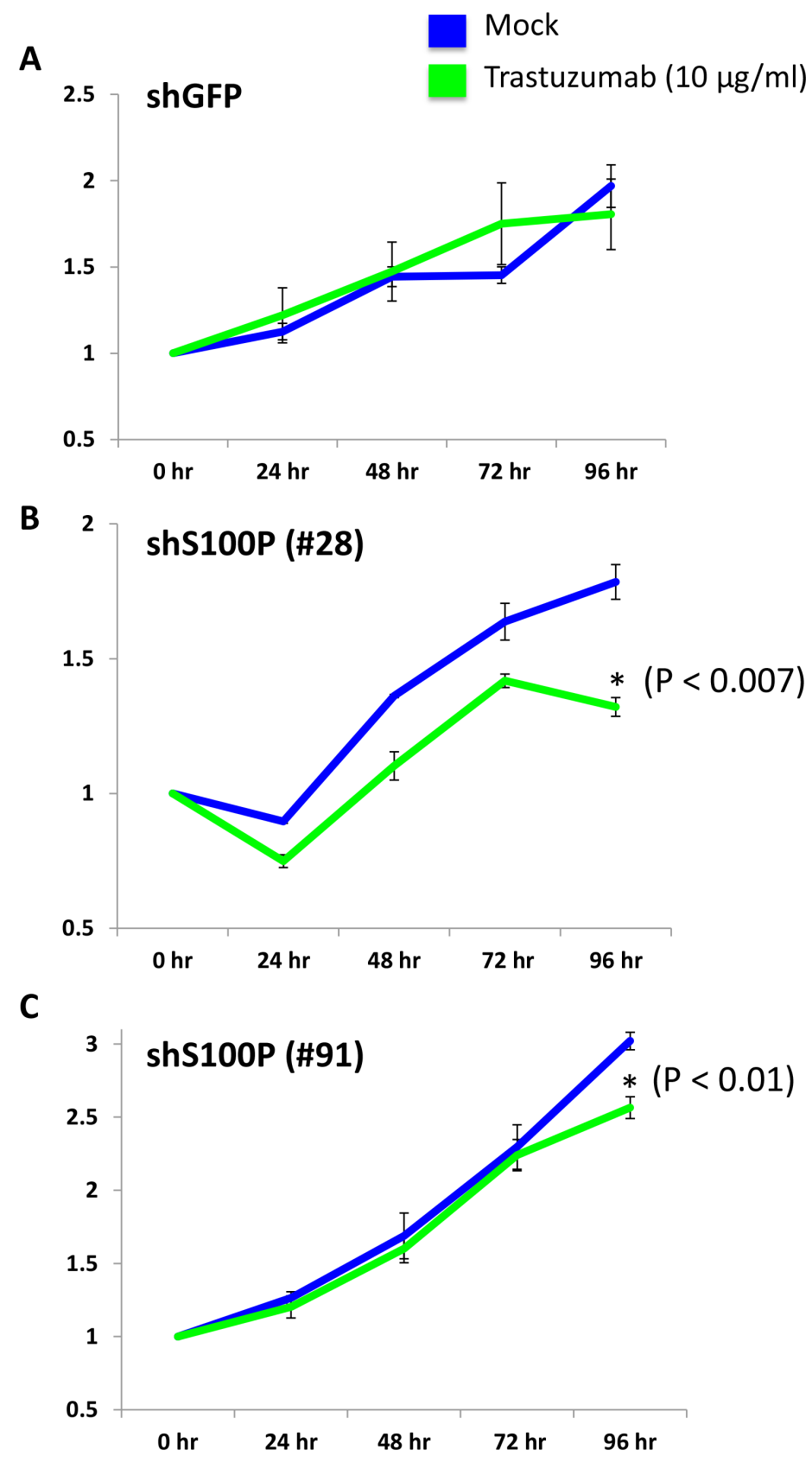

Figure 6: Knock down of S100P partially reverses TzR in cell culture. Cell proliferation analysis using MTS colorimetric assay demonstrated that TzR BT474 cells treated with either mock vs trastuzumab $(10 \mu \mathrm{g} / \mathrm{ml})$ for 96 hours show decreased proliferation rate when S100P levels are depleted with two independent shRNAs targeting S100P vs a negative control shRNA targeting GFP. 
provides a mechanism for activating Ras/MEK/MAPK independent of HER2 signaling in TzR cells.

\section{Upregulation of S100P is driven by epigenetic changes at enhancers}

To identify the potential mechanism(s) that drive increased S100P expression in TzR tumors and cell lines, we examined global changes in the epigenome at enhancer regions. Specifically, we examined changes in histone $\mathrm{H} 3$ lysine 4 monomethylation (H3K4me1) and $\mathrm{H} 3$ lysine 27 acetylation (H3K27ac), two key histone modifications associated with active enhancers [15]. First, we identified genome-wide changes in $\mathrm{H} 3 \mathrm{~K} 4 \mathrm{mel}$ and $\mathrm{H} 3 \mathrm{~K} 27 \mathrm{ac}$ between TzS and TzR cells (Supplementary Figure S5, Supplementary Files S8 and S9). Next, we specifically examined enhancers surrounding top candidate genes identified in our RNA-seq analysis, including S100P (Figure 8). Both H3K4me1 and H3K27ac profiles indicate that the upregulation of S100P in TzR cells is driven by epigenetic changes at several enhancers regulating S100P (Figure 8, and Supplementary Figure S6). These findings indicate that trastuzumab-resistance is wired at the epigenetic level, at least for a subset of genes identified in our study.

\section{DISCUSSION}

Trastuzumab was originally developed and utilized as an adjuvant therapy for HER2-positive metastatic breast cancer, and currently as an adjuvant therapy in early-stage HER2-positive patients [16-19]. The clinical utilization of trastuzumab has revolutionized the treatment of HER2positive breast cancer patients, however, a significant

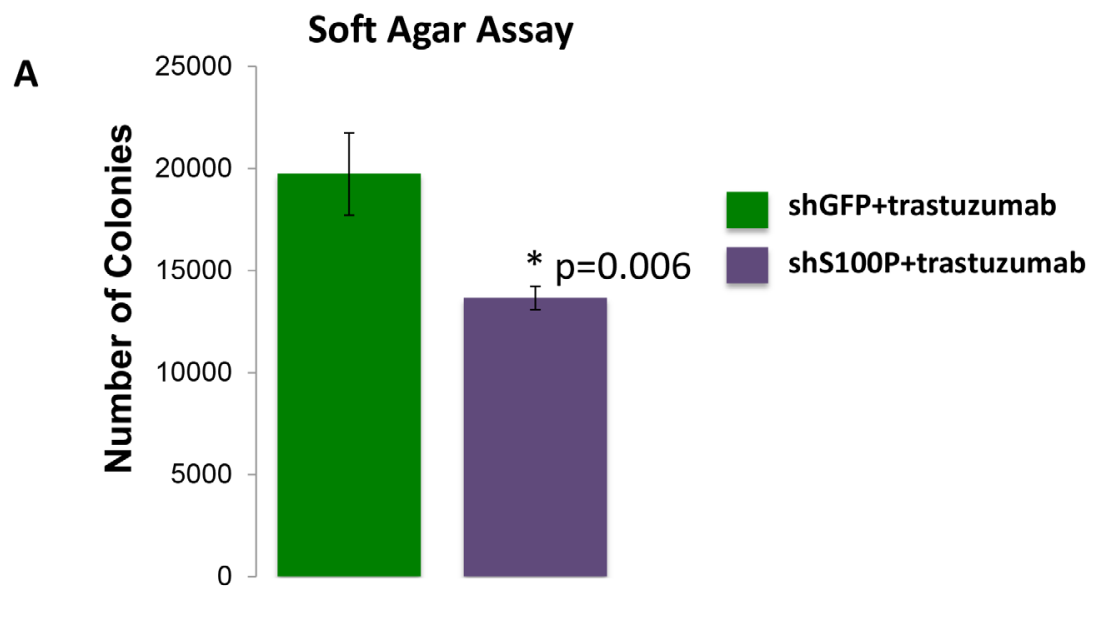

\section{BT474 (TzS)}

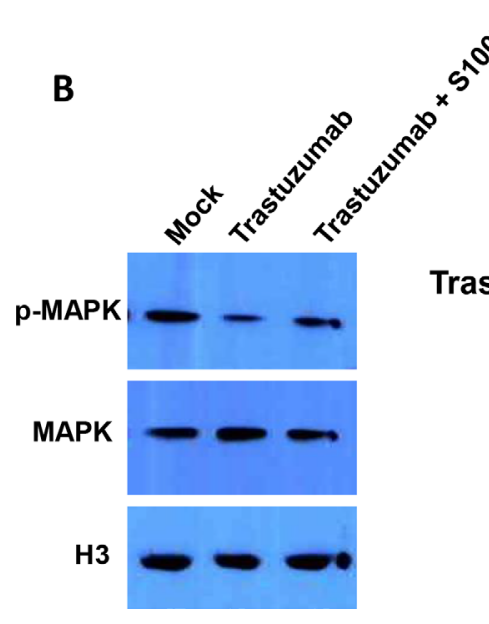

BT474 (TzR)

C

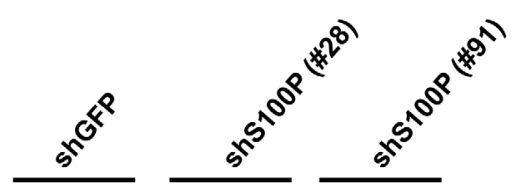

Trastuzumab:

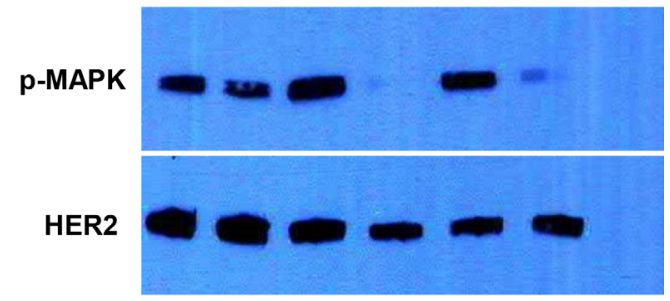

Figure 7: S100P activates Ras/MEK/MAPK pathway. (A) BT474 cells infected with shS100P lentivirus or control shGFP lentivirus were plated into soft agar to assess anchorage independent growth (AIG). Cells were grown in the presence of $10 \mu \mathrm{g} / \mathrm{mL}$ Trastuzumab for 3 weeks. ( $P<0.01$, Student's $t$ test). (B) Trastuzumab treatment inhibits p-MAPK levels in TzS BT474 cells, but the addition of recombinant S100P protein is sufficient to restore p-MAPK levels. (C) Knock down of S100P in TzR cells results in significant decrease in p-MAPK levels when cells are treated with trastuzumab, further supporting a role of S100P in trastuzumab-resistance. 
percentage of patients $(\sim 30 \%)$ do not respond to trastuzumab or acquire resistance to it, by largely unknown mechanisms $[9,10]$. Because of the immense therapeutic benefits of trastuzumab to HER2-positive breast cancer patients, it is critical to identify the mechanisms of TzR. Although a number of models of TzR have been proposed, none has been proven to be present in clinical specimens from patients treated with Trastuzmab. In this manuscript,

\section{Ratio of H3K4me1: BT474_TzR vs BT474_TzS}

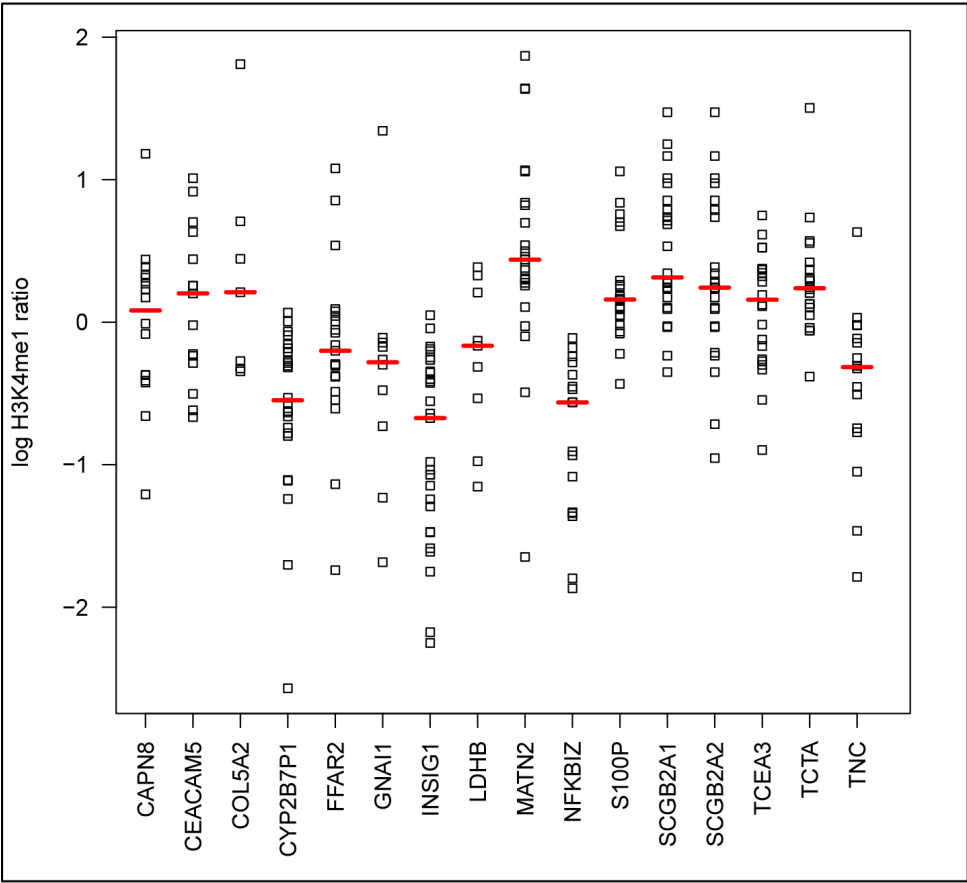

Ratio of H3K27ac: BT474_TzR vs BT474_TzS

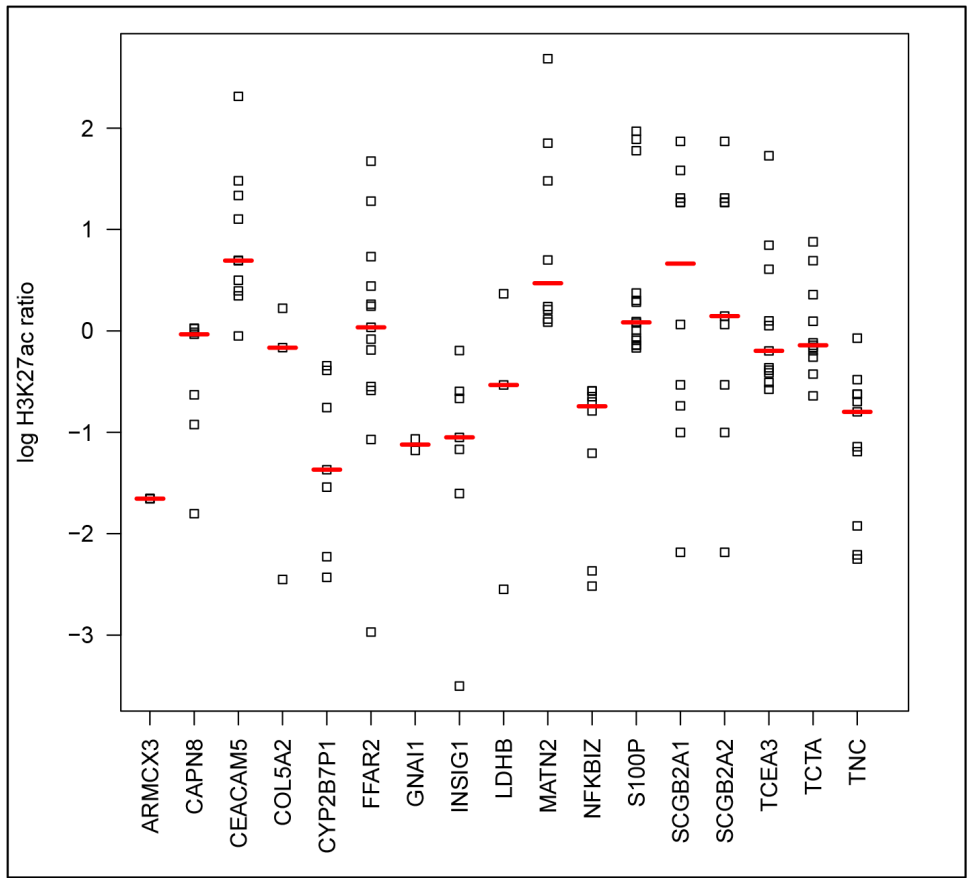

Figure 8: S100P upregulation in TzR cells is wired at the epigenetic level. All high confidence H3K4me1 and H3K27ac peaks (MACS, $p$-value $<1 \mathrm{E}-9$ ) within $150 \mathrm{~KB}$ of the TSS for top genes associated with trastuzumab-resistance were retrieved and plotted as log2 ratio of the ChIP signal in TzR BT474 vs TzS BT474 cell line. Red lines demarcate the median signal fold change for each gene. (top panel) Each data point corresponds to relative levels of H3K4me1 ChIP-seq signals (trastuzumab-resistant/ trastuzumab-sensitive) located within $150-\mathrm{kb}$ of each of the 16 dysregulated genes. (bottom panel) Same as top, but for H3K27ac. 
we have taken an unbiased genome-wide approach to identify genes and pathways that may drive and/or contribute to TzR. By utilizing next generation RNA-seq (RNA-seq) of RNA isolated from tumors and cell lines that are either TzS or TzR, we identified key genes and pathways that potentially contribute to TzR. Importantly, the utilization of tumor biopsies from patients that are either sensitive or resistant to trastuzumab enabled us to obtain clinically relevant data. However, it is important to point out that the tissue culture models used in our studies do not represent all HER2+ tumors, and thus, we may have missed other key genes and pathways that could also contribute to trastuzumab-resistance.

Many of the mRNAs that we identified as associated with TzR have been previously shown to be dysregulated in various types of cancer. MATN2 and SCGB2A2 are upregulated in ovarian cancer [20, 21]; S100P is upregulated in prostate cancer, invasive ductal carcinomas, and pancreatic cancer [22]; LDHB is a biomarker for triple negative breast cancer [23]; and TNC is associated with Tamoxifen resistance in breast cancer [24]. Although the exact roles of these genes in TzR are yet to be elucidated, previous studies of these genes in cancer should provide some preliminary insights. For example, the role of S100P in the activation of RAGE-mediated Ras/MEK/MAPK signaling provides clues into its role in TzR [14, 25].

S100P emerged as a top candidate for functional studies as it showed strong association with TzR both in vivo and in vitro studies, it is highly upregulated in many cancer types, and it belongs to the S100 family of proteins with well-documented roles in tumorigenesis [13]. S100 family members, including S100P, function as homodimers with a $\mathrm{Ca}^{2+}$-binding EF-hand motif [26]. Calcium binding to S100P promotes a conformational change exposing hydrophobic residues, which enables the interaction with protein binding partners [27]. S100P acts as both an intracellular and extracellular signaling molecule, and has been observed in the nucleus, cytoplasm, and extracellular matrix [28]. Previous studies of S100P in breast cancer have demonstrated that the expression of S100P correlates with breast cancer progression and decreased patient survival [29-32]. Also, S100P roles in tumorigenesis have been well-documented in pancreatic cancer. These studies have demonstrated that S100P exerts its oncogenic activities via interaction and activation of the receptor RAGE [14, 25]. The extracellular ligand-binding domain of RAGE is known to bind to a number of ligands, including $\mathrm{S} 100 \mathrm{P}$, to initiate downstream signaling pathways that promote cell proliferation, survival, and motility [33, 34]. Blocking S100P interaction with RAGE is sufficient to significantly impact the growth of tumors $[25,33]$. These findings suggest that S100PRAGE interaction could be therapeutically blocked to reverse TzR, at least in a subset of HER2+ patients.
To gain insights into the potential mechanisms driving changes in gene expression of S100P and other key genes identified in our study in TzS vs TzR cells, we examined chromatin changes at enhancers. Enhancers play major roles in regulating gene expression in virtually all cell types, and are known to become altered in cancer [15]. Two key chromatin marks, H3K4me1 and H3K27ac, identify active enhancers across the genome, and examining these two marks in TzS vs TzR cells demonstrated changes in enhancer activity at many loci. Enhancers regulating S100P showed higher levels of both H3K4me1 and H3K27ac in TzR cells as compared to $\mathrm{TzS}$ cells. These observations suggest that TzR cells acquire epigenetic changes that impact gene expression, and consequently, growth advantages.

Our study also led to the identification of long intergenic non-coding RNAs (lincRNAs) that are dysregulated in TzR tumors and cell lines. We and others previously demonstrated critical roles of lincRNAs in regulating gene expression at the transcriptional and posttranscriptional level in mammalian and other systems [35-44]. Currently, the role of these lincRNAs in TzR is yet to be elucidated, but they could potentially offer novel mechanistic insights and therapeutic targets in cancer biology. A major mechanism utilized by lincRNAs to regulate gene expression is via the recruitment of chromatin-modifying complexes and other proteins to specific gene loci $[38,42,43,45-50]$. Thus, such lincRNAs, when they become dysregulated in cancer, could have profound effect on the epigenome [42]. By targeting such lincRNAs, we could potentially modulate the epigenetic landscape of cancer cells to a normal state, at least partially [51]. A key advantage of targeting lincRNAs is their tissue-specific expression, potentially reducing negative side effects. In future studies, we will assess the roles of these lincRNAs in TzR, and their potential utility as therapeutic targets.

In summary, we have utilized both clinical HER2+ tumor samples and cell culture models of trastuzumabresistance to identify key mRNAs and lincRNAs associated with resistance to trastuzumab in tumors in vivo. Our findings provide a small list of potential therapeutic targets that can be experimentally tested to develop novel therapies for this aggressive form of breast cancer.

\section{MATERIALS AND METHODS}

\section{Clinical trial information}

Brown University Oncology Group (Brown University, Yale University, Cedar-Sinai Center), PI: William Sikov MD; Correlative Science PI: Lyndsay Harris MD; BrUOG Study ID: BR-211B; Clinical Trials. gov ID NCT00617942. 


\section{RNA isolation and next generation RNA sequencing (RNA-seq) of clinical trial tumor samples}

Patient biopsies cores were flash frozen for processing. RNA was isolated from samples using AllPrep (Qiagen) and amplified using Ovation RNA-seq System (NuGen). Library preparation of samples was completed using TruSeq v3 (Illumina) and sequenced on the Illumina HiSeq 2500 platform. Cufflinks V2.0.2 was used to annotate the aligned reads to human mRNA and lincRNA. Expression values were calculated as FPKM (fragment per kilobase of exon per million of mapped fragments). For our analysis, transcript expression was defined as if the FPKM value across either all non-responders (TzR) or all responders (TzS) samples were $\geq 1.0$ for mRNAs and $\geq 0.25$ for lincRNAs. The mean expression was calculated for $\mathrm{TzR}$ and $\mathrm{TzS}$ and the fold change was calculated as $\mathrm{TzR} / \mathrm{TzS}$ to identify differentially expressed transcripts. Transcripts were defined as differentially expressed if the fold change was $\geq 2.0$ or $\leq 0.5$. Heatmaps were generated in $\mathrm{R}$ using heatmap. 2 with $\mathrm{Z}$ scores scaled by row using standard $\mathrm{Z}$ score calculation of log fold change.

\section{Cell lines}

TzS cells were treated with $10 \mu \mathrm{g} / \mathrm{ml}$ trastuzumab for at least six weeks to acquire TzR. Human breast cancer cell lines used in this study were grown in Hybri-Care Medium (ATCC_46-X ${ }^{\mathrm{TM}}$ ) supplemented with 10\% fetal bovine serum (Bioexpress) and 100 units $/ \mathrm{ml}$ penicillin and $100 \mu \mathrm{g} / \mathrm{ml}$ streptomycin (Life Technologies) at $37^{\circ} \mathrm{C}$ with $5 \% \mathrm{CO}_{2}$. TzR cell lines are maintained in $10 \mu \mathrm{g} / \mathrm{ml}$ of trastuzumab.

\section{Cell proliferation assay}

Cell proliferation assays were performed using CellTiter $96^{\circledR} \mathrm{AQ}_{\text {ueous }}$ One Solution Cell Proliferation Assay (Promega) as indicated by manufacturer's instructions and read as absorbance at $490 \mathrm{~nm}$.

\section{Western blot analyses}

Protein lysates were prepared with Laemmli Sample Buffer (BioRad) and separated on a 4-20\% gradient SDS-PAGE Mini-Protean TGX ${ }^{\mathrm{TM}}$ Gels (BioRad). Gels were transferred to nitrocellulose membrane (Thermo Scientific) and probed with primary antibodies overnight at the following dilutions: anti-actin (Ambion, AM4302, $3.1 \mathrm{mg} / \mathrm{ml}$ ) 1:1000, anti-HER2/Erb2 (Cell Signaling, 2242S) 1:1000, anti-HER2-phospho (Fisher, MS-1072-P0, $200 \mu \mathrm{g} / \mathrm{ml})$ 1:500, anti-p44/42 MAPK (Erk1/2) (3A7) (Cell Signaling, 9107S) 1:1000, anti-phospho-p44/42 MAPK (Erk1/2) (Thr202/Tyr204) (Cell Signaling, 4370P) 1:500, and anti-H3 (Cell Signaling, 9715S) 1:2000. Secondary antibodies anti-mouse HRP (Thermo Scientific, 32230) and anti-rabbit HRP (Abcam, ab6721) were used at 1:10,000 dilutions. HRP was activated using SuperSignal West Pico Chemiluminescent Substrate (Thermo Scientific) for autoradiography.

\section{RNA isolation from cell lines}

RNA was isolated from cell lines using RNeasy ${ }^{\circledast}$ Mini Kit (Qiagen) according to the manufacturer's protocol with an addition of DNase (Qiagen) treatment step after the first wash to remove DNA contamination.

\section{Next generation RNA-sequencing}

RNA isolated from cell lines was assessed for quality using BioRad Experion with an RNA integrity number $(\mathrm{RIN}) \geq 8$ as threshold for high quality, suitable for RNA-sequencing. Library preparation was performed using Scriptseq ${ }^{\text {TM }}$ Complete Gold (Human/Mouse/Rat) (Illumina) and sequenced on Illumina HiSeq 2500 with six samples run per flow cell. The $100 \mathrm{bp}$ paired-end strandspecific sequences were mapped to the human genome release hg19 using TopHat with 2 mismatches allowed for full-length reads. Raw reads were mapped to human mRNAs annotated in the RefSeq database and lincRNAs annotated in Cabili et al. [52] using Cufflinks V2.0.2. Expression values were calculated as FPKM (fragment per kilobase of exon per million of mapped fragments). Transcripts were considered expressed if FPKM values across either all TzR samples or all TzS samples were $\geq 1.0$ for mRNA and $\geq 0.25$ for lincRNAs. The mean expression values were calculated for expressed transcripts followed by fold change (TzR/TzS) with statistical significance calculated using a paired $t$-test. Transcripts were defined as differentially expressed if the fold change was $\geq 2.0$ or $\leq 0.5$ and $p<0.05$. Heatmaps were generated in $\mathrm{R}$ using heatmap. 2 with $\mathrm{Z}$ scores scaled by row using standard Z score calculation of log fold change.

\section{Quantitative Real-time PCR (qRT-PCR)}

RNA was converted to cDNA using RNA to cDNA EcoDry ${ }^{\mathrm{TM}}$ Premix Random Hexamers (Clontech). Primer pairs were designed using primer3 software (Untergrasser et al., 2007) with most spanning exon-exon boundaries. Maxima SyBr Green/ROX qPCR Master Mix (Thermo Scientific) was used for qRT-PCR. A comparative $\mathrm{C}_{\mathrm{T}}$ quantitation was performed with a hold stage of $50^{\circ} \mathrm{C}$ for $2 \mathrm{~min}$ and $95^{\circ} \mathrm{C}$ for $10 \mathrm{~min}$ followed by $40 \times$ cycles of $95^{\circ} \mathrm{C}$ for $15 \mathrm{sec}$ and $60^{\circ} \mathrm{C}$ for $1 \mathrm{~min}$, and finally a melt curve at $95^{\circ} \mathrm{C}$ for $15 \mathrm{sec}, 60^{\circ} \mathrm{C}$ for $1 \mathrm{~min}$, and a ramp to $95^{\circ} \mathrm{C}$ at $0.3^{\circ} \mathrm{C}$ increments. Analysis was done using the $2 \Delta \Delta \mathrm{C}_{\mathrm{T}}$ method with GAPDH as the reference gene [53].

\section{Apoptosis assay}

Cells were plated at 5000 cells/well in a 96-well plate. After 5 hours, the media was replaced and trastuzumab 
$(10 \mu \mathrm{g} / \mathrm{ml})$ was added. After 48 hours, Caspase-Glo ${ }^{\circledR} 3 / 7$ Assay (Promega, G8091) reagent was added to the wells. The plate was incubated for $1 \mathrm{hr}$ at room temperature and the luminescence was read by spectrophotometer. The luminescence reading was normalized to the mock treatment of each cell line to calculate relative apoptosis.

\section{ChIP-seq and analysis}

Cells were crosslinked, sonicated, chromatin immunoprecipitated and converted into libraries for deep sequencing. For each ChIP, $6 \mu \mathrm{g}$ of anti-Histone H3K4 monomethyl (Abcam ab8895) or anti-Histone H3K27 acetyl (Abcam ab4729) were bound to $100 \mu$ of protein $\mathrm{G}$ dynabeads (Life Technologies 10004D) and incubated with sonicated lysate from 5 million crosslinked cells. Immunoprecipitated DNA fragments were end-repaired, received an overhanging $\mathrm{A}$ base, and then ligated to Illumina TruSeq indexed adapters [700 nM] that were annealed from oligonucleotides (IDT, HPLC-purified). Sample clean-up between steps was performed with PCRCleanDx beads (Aline Biosciences). Adaptermodified DNA fragments were PCR-amplified and sizeselected on agarose gel for 250-350 bp. ChIP-seq libraries were sequenced on the HiSeq 2500 platform at the Case Western Reserve University Genomics Core Facility. The FASTX-Toolkit 0.0.13 (http://hannonlab.cshl.edu/ fastx_toolkit/) was used to remove adapter sequences and trim read ends using a quality score cutoff of 20. ChIP-seq data were aligned to the hg19 genome assembly (retrieved from http://cufflinks.cbcb.umd.edu/igenomes.html), using Bowtie v0.12.9 [54], allowing reads with $\leq 2$ mismatches and discarding reads with $>1$ reportable alignment ("-m 1" parameter). PCR duplicates were removed using SAMtools. Peaks were detected with MACS v1.4 with a threshold for significant enrichment of $P<1 \mathrm{E}-9$. Wiggle tracks stepped at 25 bp were generated by MACS, normalized to the mean whole-genome WIG signal and visualized on the UCSC Genome Browser.

\section{Soft agar assays}

50,000 BT474 shS100P or BT474 shGFP cells were suspended in $0.6 \%$ type VII agarose (Sigma-Aldrich) in $10 \%$ FBS RPMI medium and plated onto a bottom layer of $1.2 \%$ agar in $10 \%$ FBS RPMI medium. Cells were plated onto $60 \mathrm{~mm}$ plates in quadruplicate. $1 \mathrm{~mL}$ of $10 \% \mathrm{FBS}$ RPMI with $10 \mu \mathrm{g} / \mathrm{mL}$ trastuzumab was added 24 hours after plating. The medium was changed every 2 days, with fresh trastuzumab added, until cells were analyzed after 3 weeks. To quantify colonies, each plate was scanned using an automated multipanel scanning microscope and the digital images were analyzed using MetaMorph image quantification software.

\section{Abbreviations}

$\mathrm{TzS}=$ Trastuzumab-sensitive, TzR = TrastuzumabResistant

\section{ACKNOWLEDGMENTS}

We would like to thank Jenny Parvani and Kristy Miskimen for discussion of the results. This work was supported by the Genomics Core Facility of the CWRU School of Medicine's Genetics and Genome Sciences Department.

\section{CONFLICTS OF INTEREST}

All authors declare that there are no conflicts of interest to report.

\section{FUNDING}

for this work was supported by new investigator start-up funds to Dr. Ahmad Khalil, Case Comprehensive Cancer Center (P30 CA043703) to Dr Cheryl Thompson, BCRF grant to Dr. Lyndsay Harris, National Institutes of Health grants R01CA160356 and R01CA193677 to Peter Scacheri and a core utilization award to Dr Ahmad Khalil by the Clinical and Translational Science Collaborative of Cleveland, UL1TR000439 from the National Center for Advancing Translational Sciences (NCATS).

\section{REFERENCES}

1. Mitri Z, Constantine T, O'Regan R. The HER2 Receptor in Breast Cancer: Pathophysiology, Clinical Use, and New Advances in Therapy. Chemotherapy research and practice. 2012; 2012:743193.

2. Cancer Genome Atlas N. Comprehensive molecular portraits of human breast tumours. Nature. 2012; 490:61-70.

3. Perou CM, Sorlie T, Eisen MB, van de Rijn M, Jeffrey SS, Rees CA, Pollack JR, Ross DT, Johnsen H, Akslen LA, Fluge O, Pergamenschikov A, Williams C, et al. Molecular portraits of human breast tumours. Nature. 2000; 406:747-752.

4. Arteaga CL, Engelman JA. ERBB receptors: from oncogene discovery to basic science to mechanism-based cancer therapeutics. Cancer Cell. 2014; 25:282-303.

5. Merry CR, McMahon S, Thompson CL, Miskimen KL, Harris LN, Khalil AM. Integrative transcriptome-wide analyses reveal critical HER2-regulated mRNAs and lincRNAs in HER2+ breast cancer. Breast Cancer Res Treat. 2015; 150:321-334.

6. Sendur MA, Aksoy S, Ozdemir NY, Zengin N, Altundag K. What is the Mechanism of Progression with Trastuzumab 
Treatment - Escape or Resistance? Asian Pacific journal of cancer prevention. 2012; 13:5929-5930.

7. Oliveras-Ferraros C, Vazquez-Martin A, Martin-Castillo B, Perez-Martinez MC, Cufi S, Del Barco S, Bernado L, Brunet J, Lopez-Bonet E, Menendez JA. Pathway-focused proteomic signatures in HER2-overexpressing breast cancer with a basal-like phenotype: new insights into de novo resistance to trastuzumab (Herceptin). Int J Oncol. 2010; 37:669-678.

8. Figueroa-Magalhaes MC, Jelovac D, Connolly RM, Wolff AC. Treatment of HER2-positive breast cancer. Breast. 2014; 23:128-36.

9. Baretta Z, Guindalini RS, Khramtsova G, Olopade OI. Resistance to trastuzumab in HER2-positive mucinous invasive ductal breast carcinoma. Clinical breast cancer. 2013; 13:156-158.

10. Vu T, Claret FX. Trastuzumab: updated mechanisms of action and resistance in breast cancer. Front Oncol. 2012; 2:62.

11. Baselga J, Gelmon KA, Verma S, Wardley A, Conte P, Miles D, Bianchi G, Cortes J, McNally VA, Ross GA, Fumoleau P, Gianni L. Phase II trial of pertuzumab and trastuzumab in patients with human epidermal growth factor receptor 2-positive metastatic breast cancer that progressed during prior trastuzumab therapy. J Clin Oncol. 2010; 28:1138-1144.

12. Morris KV, Mattick JS. The rise of regulatory RNA. Nat Rev Genet. 2014; 15:423-437.

13. Bresnick AR, Weber DJ, Zimmer DB. S100 proteins in cancer. Nat Rev Cancer. 2015; 15:96-109.

14. Xie J, Mendez JD, Mendez-Valenzuela V, AguilarHernandez MM. Cellular signalling of the receptor for advanced glycation end products (RAGE). Cell Signal. 2013; 25:2185-2197.

15. Akhtar-Zaidi B, Cowper-Sal-lari R, Corradin O, Saiakhova A, Bartels CF, Balasubramanian D, Myeroff L, Lutterbaugh J, Jarrar A, Kalady MF, Willis J, Moore JH, Tesar PJ, et al. Epigenomic enhancer profiling defines a signature of colon cancer. Science. 2012; 336:736-739.

16. Slamon D, Eiermann W, Robert N, Pienkowski T, Martin M, Press M, Mackey J, Glaspy J, Chan A, Pawlicki M, Pinter T, Valero V, Liu MC, et al. Adjuvant trastuzumab in HER2positive breast cancer. N Engl J Med. 2011; 365:1273-1283.

17. Perez EA, Romond EH, Suman VJ, Jeong JH, Davidson NE, Geyer CE, Jr., Martino S, Mamounas EP, Kaufman PA, Wolmark N. Four-year follow-up of trastuzumab plus adjuvant chemotherapy for operable human epidermal growth factor receptor 2-positive breast cancer: joint analysis of data from NCCTG N9831 and NSABP B-31. J Clin Oncol. 2011; 29:3366-3373.

18. Slamon DJ, Leyland-Jones B, Shak S, Fuchs H, Paton V, Bajamonde A, Fleming T, Eiermann W, Wolter J, Pegram M, Baselga J, Norton L. Use of chemotherapy plus a monoclonal antibody against HER2 for metastatic breast cancer that overexpresses HER2. N Engl J Med. 2001; 344:783-792.
19. Brufsky AM. Current Approaches and Emerging Directions in HER2-resistant Breast Cancer. Breast Cancer (Auckl). 2014; 8:109-118.

20. Januchowski R, Zawierucha P, Rucinski M, Zabel M. Microarray-based detection and expression analysis of extracellular matrix proteins in drugresistant ovarian cancer cell lines. Oncol Rep. 2014; 32:1981-1990.

21. Fischer K, von Brunneck AC, Hornung D, Denkert C, Ufer C, Schiebel H, Kuhn H, Borchert A. Differential expression of secretoglobins in normal ovary and in ovarian carcinoma-overexpression of mammaglobin-1 is linked to tumor progression. Arch Biochem Biophys. 2014; 547:27-36.

22. Arumugam T, Simeone DM, Van Golen K, Logsdon CD. S100P promotes pancreatic cancer growth, survival, and invasion. Clin Cancer Res. 2005; 11:5356-5364.

23. Lehmann BD, Pietenpol JA. Identification and use of biomarkers in treatment strategies for triple-negative breast cancer subtypes. J Pathol. 2014; 232:142-150.

24. Helleman J, Jansen MP, Ruigrok-Ritstier K, van Staveren IL, Look MP, Meijer-van Gelder ME, Sieuwerts AM, Klijn JG, Sleijfer S, Foekens JA, Berns EM. Association of an extracellular matrix gene cluster with breast cancer prognosis and endocrine therapy response. Clin Cancer Res. 2008; 14:5555-5564.

25. Arumugam T, Ramachandran V, Logsdon CD. Effect of cromolyn on S100P interactions with RAGE and pancreatic cancer growth and invasion in mouse models. J Natl Cancer Inst. 2006; 98:1806-1818.

26. Donato R. S100: a multigenic family of calcium-modulated proteins of the EF-hand type with intracellular and extracellular functional roles. Int J Biochem Cell Biol. 2001; 33:637-668.

27. Schafer BW, Wicki R, Engelkamp D, Mattei MG, Heizmann CW. Isolation of a YAC clone covering a cluster of nine S100 genes on human chromosome 1q21: rationale for a new nomenclature of the S100 calcium-binding protein family. Genomics. 1995; 25:638-643.

28. Jiang H, Hu H, Tong X, Jiang Q, Zhu H, Zhang S. Calciumbinding protein $\mathrm{S} 100 \mathrm{P}$ and cancer: mechanisms and clinical relevance. J Cancer Res Clin Oncol. 2012; 138:1-9.

29. Guerreiro Da Silva ID, Hu YF, Russo IH, Ao X, Salicioni AM, Yang X, Russo J. S100P calcium-binding protein overexpression is associated with immortalization of human breast epithelial cells in vitro and early stages of breast cancer development in vivo. Int J Oncol. 2000; 16:231-240.

30. Mackay A, Jones C, Dexter T, Silva RL, Bulmer K, Jones A, Simpson P, Harris RA, Jat PS, Neville AM, Reis LF, Lakhani SR, O'Hare MJ. cDNA microarray analysis of genes associated with ERBB2 (HER2/neu) overexpression in human mammary luminal epithelial cells. Oncogene. 2003; 22:2680-2688.

31. Schor AP, Carvalho FM, Kemp C, Silva ID, Russo J. S100P calcium-binding protein expression is associated with high-risk proliferative lesions of the breast. Oncol Rep. 2006; 15:3-6. 
32. Wang G, Platt-Higgins A, Carroll J, de Silva Rudland S, Winstanley J, Barraclough R, Rudland PS. Induction of metastasis by S100P in a rat mammary model and its association with poor survival of breast cancer patients. Cancer Res. 2006; 66:1199-1207.

33. Taguchi A, Blood DC, del Toro G, Canet A, Lee DC, Qu W, Tanji N, Lu Y, Lalla E, Fu C, Hofmann MA, Kislinger T, Ingram $\mathrm{M}$, et al. Blockade of RAGE-amphoterin signalling suppresses tumour growth and metastases. Nature. 2000; 405:354-360.

34. Kierdorf K, Fritz G. RAGE regulation and signaling in inflammation and beyond. J Leukoc Biol. 2013; 94:55-68.

35. Xing Z, Lin A, Li C, Liang K, Wang S, Liu Y, Park PK, Qin L, Wei Y, Hawke DH, Hung MC, Lin C, Yang L. lncRNA directs cooperative epigenetic regulation downstream of chemokine signals. Cell. 2014; 159:1110-1125.

36. Hou P, Zhao Y, Li Z, Yao R, Ma M, Gao Y, Zhao L, Zhang Y, Huang B, Lu J. LincRNA-ROR induces epithelialto-mesenchymal transition and contributes to breast cancer tumorigenesis and metastasis. Cell death \& disease. 2014; 5:e1287.

37. Grote P, Wittler L, Hendrix D, Koch F, Wahrisch S, Beisaw A, Macura K, Blass G, Kellis M, Werber M, Herrmann BG. The tissue-specific lncRNA Fendrr is an essential regulator of heart and body wall development in the mouse. Dev Cell. 2013; 24:206-214.

38. Gomez JA, Wapinski OL, Yang YW, Bureau JF, Gopinath S, Monack DM, Chang HY, Brahic M, Kirkegaard K. The NeST long ncRNA controls microbial susceptibility and epigenetic activation of the interferon-gamma locus. Cell. 2013; 152:743-754.

39. Moran VA, Perera RJ, Khalil AM. Emerging functional and mechanistic paradigms of mammalian long non-coding RNAs. Nucleic Acids Res. 2012; 40:6391-6400.

40. Carrieri C, Cimatti L, Biagioli M, Beugnet A, Zucchelli S, Fedele S, Pesce E, Ferrer I, Collavin L, Santoro C, Forrest AR, Carninci P, Biffo S, et al. Long non-coding antisense RNA controls Uchll translation through an embedded SINEB2 repeat. Nature. 2012; 491:454-457.

41. Gong C, Maquat LE. IncRNAs transactivate STAU1mediated mRNA decay by duplexing with 3' UTRs via Alu elements. Nature. 2011; 470:284-288.

42. Merry CR, Forrest ME, Sabers JN, Beard L, Gao X-H, Hatzoglou M, Jackson MW, Wang Z, Markowitz SD, Khalil AM. DNMT1-associated long non-coding RNAs regulate global gene expression and DNA methylation in colon cancer. Human Molecular Genetics. 2015; 24:6240-6253.

43. Khalil AM, Guttman M, Huarte M, Garber M, Raj A, Rivea Morales D, Thomas K, Presser A, Bernstein BE, van Oudenaarden A, Regev A, Lander ES, Rinn JL. Many human large intergenic noncoding RNAs associate with chromatin-modifying complexes and affect gene expression. Proc Natl Acad Sci U S A. 2009; 106:11667-11672.

44. Khalil AM, Faghihi MA, Modarresi F, Brothers SP, Wahlestedt C. A novel RNA transcript with antiapoptotic function is silenced in fragile $\mathrm{X}$ syndrome. PLoS One. 2008; 3:e1486.

45. Klattenhoff CA, Scheuermann JC, Surface LE, Bradley RK, Fields PA, Steinhauser ML, Ding H, Butty VL, Torrey L, Haas S, Abo R, Tabebordbar M, Lee RT, et al. Braveheart, a long noncoding RNA required for cardiovascular lineage commitment. Cell. 2013; 152:570-583.

46. Chu C, Qu K, Zhong FL, Artandi SE, Chang HY. Genomic maps of long noncoding RNA occupancy reveal principles of RNA-chromatin interactions. Mol Cell. 2011; 44:667-678.

47. Tsai MC, Manor O, Wan Y, Mosammaparast N, Wang JK, Lan F, Shi Y, Segal E, Chang HY. Long noncoding RNA as modular scaffold of histone modification complexes. Science. 2010; 329:689-693.

48. Schmitz KM, Mayer C, Postepska A, Grummt I. Interaction of noncoding RNA with the rDNA promoter mediates recruitment of DNMT3b and silencing of rRNA genes. Genes Dev. 2010; 24:2264-2269.

49. Huarte M, Guttman M, Feldser D, Garber M, Koziol MJ, Kenzelmann-Broz D, Khalil AM, Zuk O, Amit I, Rabani M, Attardi LD, Regev A, Lander ES, et al. A large intergenic noncoding RNA induced by p53 mediates global gene repression in the p53 response. Cell. 2010; 142:409-419.

50. Gupta RA, Shah N, Wang KC, Kim J, Horlings HM, Wong DJ, Tsai MC, Hung T, Argani P, Rinn JL, Wang Y, Brzoska P, Kong B, et al. Long non-coding RNA HOTAIR reprograms chromatin state to promote cancer metastasis. Nature. 2010; 464:1071-1076.

51. Moskalev EA, Schubert M, Hoheisel JD. RNA-directed epigenomic reprogramming: an emerging principle of a more targeted cancer therapy? Genes Chromosomes Cancer. 2012; 51:105-110.

52. Cabili MN, Trapnell C, Goff L, Koziol M, Tazon-Vega B, Regev A, Rinn JL. Integrative annotation of human large intergenic noncoding RNAs reveals global properties and specific subclasses. Genes Dev. 2011; 25:1915-1927.

53. Livak KJ, Schmittgen TD. Analysis of relative gene expression data using real-time quantitative PCR and the 2(-Delta Delta C(T)) Method. Methods. 2001; 25:402-408.

54. Langmead B, Trapnell C, Pop M, Salzberg SL. Ultrafast and memory-efficient alignment of short DNA sequences to the human genome. Genome Biol. 2009; 10:R25. 